\title{
Orientaciones competitivas culturales en la empresa familiar
}

\author{
Antonio José CARRASCO HERNÁNDEZ \\ Departamento de Organización de Empresa y Finanzas \\ Universidad de Murcia \\ antonioc@um.es \\ Gregorio SÁNCHEZ MARIN \\ Departamento de Organización de Empresa y Finanzas \\ Universidad de Murcia \\ gresanma@um.es \\ Ignacio DANVILA DEL VALLE \\ Departamento de Organización de Empresas \\ Universidad Complutense de Madrid \\ idanvila@ucm.es \\ Miguel Ángel SASTRE CASTILLO \\ Departamento de Organización de Empresas \\ Universidad Complutense de Madrid \\ Profesor Visitante del Instituto Tecnológico Autónomo de México ITAM \\ masastre@ucm.es
}

Recibido: 20-07-2015

Aceptado: 15-11-2015

\section{RESUMEN}

Este trabajo discute acerca de cómo se configuran los recursos estratégicos culturales en la empresa familiar, en un intento de entender mejor las diferencias y similitudes entre empresas familiares y no familiares. Para ello, el marco conceptual empleado es el instrumento de valoración de la cultura de Cameron y Quinn (1999), en el que se distinguen 
cuatro tipos de orientaciones competitivas culturales (mercado, jerarquía, clan y adhocracia). Con ello se propone que la cultura organizativa varía en función del carácter de la empresa, atendiendo al grado de propiedad y dirección en manos de la familia. En concreto, la cultura clan y adhocrática caracterizan mejor a la cultura de las empresas de propiedad y dirección familiar. Mientras que la de mercado es la cultura que mejor caracteriza a las empresas no familiares. En el caso de las empresas familiares profesionalizadas, no existen diferencias culturales con respecto a las empresas de propiedad y dirección familiar.

Palabras clave: cultura organizativa, empresa familiar, teoría de recursos, capacidades.

\title{
Competitive cultural orientation in the family business
}

\begin{abstract}
This paper analyses the make-up of strategic cultural resources in the family business to establish important declarations which can define better the differences and similarities between family and non family businesses. The tool used here is the culture valuation tool proposed by Cameron and Quinn (1999) which identifies four types of cultural, competitive foci (market, hierarchy, clan and adhocracy). The organizational culture varies according to the nature of the business in terms of the degree of family ownership and management. Specifically, the clan and adhocracy culture are what best characterize the culture of family owned and managed businesses, while it is the market culture which best characterizes nonfamily businesses. Professionalized family businesses show no cultural differences with respect to family owned and managed firms.
\end{abstract}

Keywords: organizational culture, family business, resource based view.

Sumario: 1. Introducción 2. La cultura organizativa en la empresa familiar: una perspectiva de recursos y capacidades 3 . Conclusiones.

\section{INTRODUCCIÓN}

Muchos trabajos han adoptado la teoría de recursos y capacidades como marco teórico apropiado para caracterizar la competitividad de la empresa desde sus recursos (Barney, 1986). Dicha teoría ha ayudado a mejorar la comprensión de las organizaciones y de sus recursos desde un escenario competitivo. No obstante, y a pesar de los numerosos trabajos existentes, el estudio de cada uno de los recursos organizativos no ha sido abordado con el mismo rigor académico. Este es el caso de la cultura organizativa, la cual, a pesar de ser un importante recurso estratégico para desarrollar ventajas competitivas frente a la competencia (Zahra et al., 2004; Barney, 1986) y mejorar la eficiencia (Denison, 1990; Kotter y Heskett, 1992) es un aspecto aún por examinar en profundidad.

Este análisis resulta aún más interesante, si cabe, en el ámbito de la empresa familiar, en la que aparecen un conjunto de valores, creencias e intereses que la 
diferencian de cualquier otro tipo de organización (Dyer, 1986), al estar altamente influenciada por los vínculos de la familia en la empresa (ver Gómez-Mejía et al., 2001). Y no sólo la presencia de valores, creencias e intereses distintos hace importante su estudio, sino también el papel que estos juegan en el éxito de la empresa familiar, donde los lazos de parentesco familiar, exclusivos en tales empresas, la conducen a una situación más exitosa en el mercado (Barontini y Caprio, 2006).

La literatura de empresa familiar ha analizado la influencia de diferentes factores en la cultura organizativa. Así, Zahra et al. (2004), Rogoff y Heck (2003), Hall et al. (2001) y Pistrui et al. (2001) han mostrado su conexión con la actividad emprendedora. Poza et al. (1997) su relación con los valores del fundador en la cultura organizativa de la empresa familiar. Sorenson (2000) su nexo con el estilo de liderazgo y el éxito de la empresa. Y Chrisman et al. (2002), Steier (2001), Pistrui et al. (2001) y Corbetta y Montemerlo (1999) su vínculo con la historia de la empresa y la cultura del entorno. Así, esta multiplicidad de factores, que influyen sobre la cultura de la empresa familiar, y su carácter intangible en la empresa, la convierten en un recurso diferenciador, distintivo y difícil de imitar por otras organizaciones (Zahra et al., 2004).

Sin embargo, pocos estudios se han centrado en la caracterización de cultura organizativa de la empresa familiar en sí misma (Denison et al., 2004; Zahra et al., 2004; Hall et al., 2001; Dyer, 1986). Además, tales estudios previos fallan al distinguir a las empresas familiares de las no familiares (Zahra et al., 2004), por lo que los resultados empíricos que se han encontrado hasta el momento no son concluyentes. Y, de ellos, tan sólo Zahra et al. (2004) la examinan integralmente desde la perspectiva de los recursos y capacidades.

Los argumentos de recursos y capacidades para la cultura organizativa se basan, como sugiere Barney (1986), en la idea de que la heterogeneidad de desempeños entre empresas puede explicarse a través de la capacidad de cada una de ellas para obtener ventajas sobre la competencia, así como de la habilidad para identificar, desarrollar, proteger y aprovechar los activos estratégicos empresariales, en este caso, a través de la cultura organizativa y, así, alcanzar ventajas competitivas sostenibles. Para realizar este análisis general caracterizador, el modelo de valores competitivos (Competing Value Framework - CVF) de Cameron y Quinn (1999), en el que se distinguen cuatro escenarios competitivos culturales - clan, adhocracia, mercado y jerarquía -, permite examinar la cultura organizativa de la empresa familiar desde un enfoque estratégico y competitivo.

Así, son dos los objetivos principales de este trabajo. En primer lugar, examinar desde la teoría de recursos y capacidades la relación entre dichos escenarios competitivos de la cultura y el carácter familiar de la empresa, esto es, explicar el posicionamiento de la empresa en los distintos escenarios culturales en función de su estructura de propiedad y de dirección. Y en segundo lugar, discutir si el 
carácter familiar de la empresa podría ejercer un efecto moderador en la relación entre cultura organizativa y resultados.

Este trabajo ofrece diversas contribuciones a la literatura sobre cultura organizativa. Primero, nos ayuda a entender mejor la cultura organizativa como recurso estratégico en la empresa familiar, en segundo lugar, extiende la literatura de recursos y capacidades a la empresa familiar para comprender como varía la cultura organizativa entre las empresas y por último, ayuda a comprender como el carácter familiar de la empresa afecta a la cultura organizativa y ésta a los resultados de la organización.

\section{LA CULTURA ORGANIZATIVA EN LA EMPRESA FAMILIAR: UNA PERSPECTIVA DE RECURSOS Y CAPACIDADES}

La tesis central de la teoría de recursos y capacidades reside en dos conceptos (Barney, 1991; Amit y Schoemaker, 1993). Por un lado, en la heterogeneidad entre las empresas, cada empresa es diferente en función de su dotación de recursos y capacidades y sobre estos se sustentan las ventajas competitivas. Por otro lado, en la sostenibilidad de las rentas, ya que la heterogeneidad puede o no persistir en el tiempo y, por lo tanto, las empresas pueden obtener rentas superiores en el largo plazo. Los recursos y capacidades que permiten alcanzar ventajas competitivas son aquellos que son valiosos y escasos (Barney, 1991; Grant, 1996) y que duran en el tiempo (Amit y Schoemaker, 1993).

Bajo esta teoría, la cultura organizativa es un recurso estratégico que genera ventajas competitivas sostenibles (Barney, 1986) a través de la transmisión de conocimientos, el riesgo asumido y la innovación (De Long y Fahey, 2000). La cultura de la empresa también es un recurso difícil de imitar (Dierickx y Cool, 1989) por la ambigüedad de sus orígenes y su relación con la historia, dinámica y evolución de la familia (Gersick et al., 1997). Se trata también de un recurso que no es sencillo de cambiar (Zahra et al., 2004).

Entendemos por cultura organizativa al conjunto de creencias, expectativas y principios fundamentales o básicos, compartidos por los miembros de una organización. Estas creencias y expectativas producen reglas de conducta (normas) que configuran poderosamente la conducta de los individuos y grupos de la organización, y de esta forma la diferencian de otras organizaciones (Alvesson, 1993). Estas creencias tienen su origen en las ideas de los fundadores, que han evolucionado a lo largo de los años hasta la actualidad a través de sus propias experiencias, las nuevas tendencias sociales y los propios valores de los directivos (Ortega Parra y Sastre Castillo, 2013).

A la cultura podemos entenderla tanto como herramienta que la dirección de la empresa puede utilizar para desarrollar ventajas competitivas que conduzcan a un mayor éxito organizativo (Alvesson, 1993), como a la propia organización en sí, entendiendo que la cultura está altamente interrelacionada con todos los recursos de la empresa, valores y supuestos subyacentes (Hall et al., 2001). Es decir, se la 
puede examinar a partir de las decisiones adoptadas en la empresa, o delimitarla en función de otros recursos organizativos.

Mientras existen numerosas dimensiones que caracterizan a la cultura organizativa y numerosos modelos para explicarla (Detert et al., 2000; Sánchez Marín et al., 2015), en este trabajo se adopta el modelo de Cameron y Quinn, un modelo general de cultura organizativa, que ajusta bien con la teoría de recursos y capacidades y que ha sido ampliamente utilizado en otros estudios (Stock et al., 2007). Estos autores se basan en el Competing Values Framework propuesto por Quinn (1988), para crear lo que denominan instrumento para la valoración de la cultura organizacional (Organizacional Cultural Assessment Instrument, OCAI). Esta herramienta es adecuada también por varios motivos (Cameron y Quinn, 1999): (1) es práctica, ya que incluye las dimensiones culturales más relevantes; (2) en ella se miden datos cuantitativos y cualitativos; (3) es manejable y fácil de aplicar; y (4) es válida, pues está siendo aplicada por un número considerable de organizaciones e investigadores.

El OCAI es una técnica que mide la cultura organizativa de la empresa sobre cuatro tipos de cultura. Los cuatro mayores modelos de cultura propuestos en la teoría organizativa (Stock et al., 2007). Estos cuatro modelos quedan definidos y reflejados en la figura 1, a partir de dos dimensiones espaciales que reflejan diferentes orientaciones de valores (Denison y Spreitzer, 1991).

\section{Figura 1}

Instrumento para la valoración de la cultura organizacional

FLEXIBILIDAD

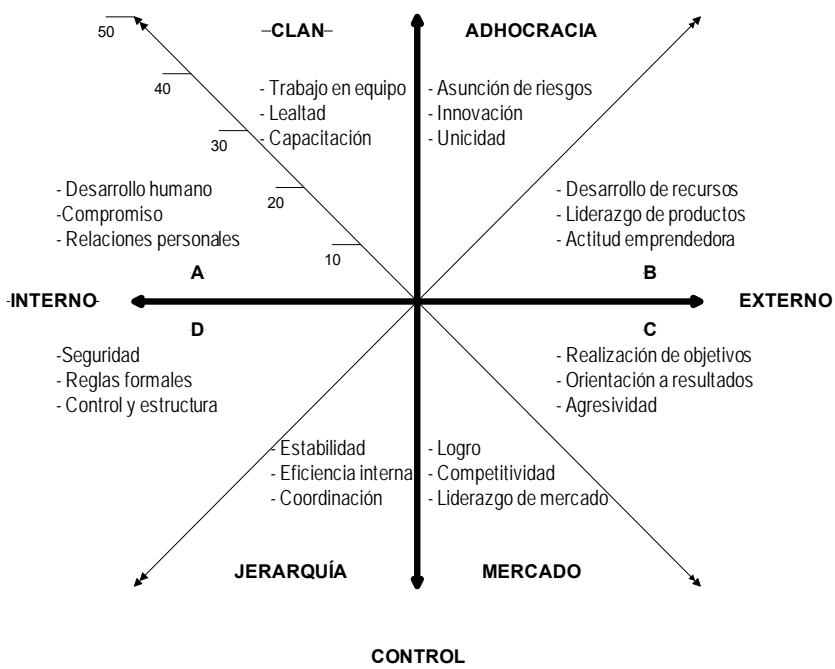

Fuente: Stock et al. (2007) 
La primera dimensión en el modelo, el eje flexibilidad-control, muestra el grado por el cual la organización enfatiza cambios o estabilidad. Una orientación a la flexibilidad refleja cambio y espontaneidad, mientras que una orientación al control refleja estabilidad, control y orden. La segunda dimensión en el modelo, el eje interno-externo, distingue entre la elección de la organización por orientarse en actividades que suceden dentro de la organización (internas) frente a las que suceden fuera, en el entorno. Una orientación interna refleja el énfasis en el mantenimiento y mejora de la organización, mientras que la orientación externa refleja el énfasis en la competitividad, adaptación e interacción con el entorno externo.

A partir de estas dos dimensiones se configuran los cuatro tipos de cultura que pueden aparecer en la empresa. La primera, la cultura clan o grupal, se corresponde con el modelo de relaciones humanas de las teoría organizativa, en el que se enfatiza la flexibilidad y el cambio y se caracteriza por unas fuertes relaciones humanas, afiliación, y una orientación en las relaciones internas organizativas. La segunda, la cultura adhocrática o de cambio, se corresponde con el modelo de sistema abierto, en el que se enfatiza la flexibilidad y la orientación al exterior o entorno. Se centra en el crecimiento, la adquisición de recursos, la creatividad y la adaptación al entorno. La tercera, la cultura racional o de mercado, se corresponde con el modelo racional de objetivos, esta externamente orientada, pero también lo está al control. Se centra en la productividad y logro, con objetivos bien definidos y competitividad externa como principal factor motivador. La cuarta, la cultura jerárquica, corresponde con el modelo de procesos internos, en el que se enfatiza la estabilidad. Sin embargo, en contraste con la cultura racional se orienta a lo interno de la organización. Esta orientación se caracteriza por la uniformidad, coordinación, eficiencia interna y preferencia por las reglas y reglamentos ${ }^{1}$.

Una asunción importante en este modelo de cultura es que cada uno de los cuatro cuadrantes representa una orientación cultural (Denison y Spreitzer, 1991). Una organización puede presentar una combinación de orientaciones culturales diferentes, es decir puede estar orientada a los cuatro tipos de cultura definidos, aunque con una restricción, y es que a mayor grado de orientación a uno de los tipos de cultura, menor grado de orientación a los otros tres, existiendo así un determinado balance entre las cuatro orientaciones culturales (Stock et al., 2007).

Por otro lado, aunque la cultura organizativa puede ser descrita a nivel general en la organización, desde argumentos de recursos y capacidades, su caracterización será mejorada y variará en función de la estructura de propiedad y dirección. Por ello, en este trabajo caracterizamos a la cultura, distinguiendo a diferentes tipos de empresas: (1) de propiedad y dirección familiar (propiedad y dirección altamente

\footnotetext{
${ }^{1}$ Estas cuatro orientaciones culturales han sido adaptadas a este modelo, desde un trabajo previo de Quinn y Spreitzer (1991).
} 
concentrada en manos de la familia la cual desea seguir vinculada al negocio en el futuro); (2) familiar profesionalizada (dirección en manos de profesionales no familiares); y (3) no familiar (propiedad muy diluida y dirección en manos de no propietarios). Además hay que considerar que los recursos en tales organizaciones son diferentes, y que las decisiones de los propietarios y directivos también varían atendiendo a sus intereses y riesgos, y al rol desempeñado en cada una de ellas.

A continuación se caracterizan las cuatro dimensiones culturales -clan, adhocracia, mercado, jerarquía- para las empresas familiares, en función del grado de presencia de la familia en la estructura de propiedad y dirección de la empresa. Para ello, considerando lo señalado anteriormente, delimitamos la cultura organizativa en la empresa familiar desde la propia organización, esto es, a partir de otros recursos y características organizativas (Hall et al., 2001), así como desde las decisiones adoptadas desde la propiedad y dirección de la empresa (Alvesson, 1993).

\section{CULTURA CLAN O GRUPAL}

Una primera dimensión de la cultura organizativa son los valores y creencias de la empresa con relación a la cultura del grupo (figura 1), esto es, unos valores orientados a la empresa y a la flexibilidad organizativa, y altamente influenciados por la familia. Valores que han caracterizado a la empresa de propiedad y dirección familiar en diferentes estudios (Gersick et al., 1997). De ahí, que autores como Gallo et al. (2004) definan a esta empresa como "aquella en la que familia y organización comparten la cultura y tradiciones parcial o totalmente y voluntariamente".

Las investigaciones realizadas en torno a la cultura en las empresas de propiedad y dirección familiar, asumen que dicha cultura está muy arraigada a la familia, consideran que la familia tiene gran influencia en ella y en sus componentes, al ser los miembros de la familia, los máximos accionistas de la empresa, y tener el poder y la posición para adoptar las principales decisiones organizativas, tanto desde la propiedad como desde la gestión organizativa. De ahí que Schulze et al. (2001) hablen de que estas organizaciones son altamente aversas al riesgo, y esta mayor aversión las conduce a ser más introvertidas, cargadas de viejas tradiciones y resistentes a los cambios del entorno (Kets de Vries, 1993; Gersick et al., 1997). Otros (Karra et al., 2006; Schulze et al., 2003) hablan incluso de una problemática peculiar en estas empresas ocasionada por sus valores altruistas, esto es, por su tendencia a favorecer determinados valores de la familia e intereses de sus miembros (habitualmente familiares) en contra de otros valores e intereses organizativos más racionales, marcados por las necesidades del mercado.

Por otro lado, el tamaño y la edad de la empresa afectan a la cultura organizativa vigente. Así, las empresas de propiedad y dirección familiar, con respecto a otras organizaciones suelen ser organizaciones más jóvenes y habitualmente tienen un menor tamaño en el mercado (Jorissen et al., 2005; Galve 
y Salas, 2003; Gersick et al., 1997), y este menor tamaño y edad, también favorece y refuerza la presencia de valores internos, que unan y definan al grupo, frente a valores externos de mercado.

Por último, son empresas en manos de un propietario-director, que adopta el papel de fundador, y sienta las bases de la cultura organizativa en la empresa (Gersick et al., 1997; Schein, 1995). Y no sólo en la etapa en la que el fundador está al mando de la empresa, que suele larga y significativamente mayor a la de cualquier directivo en las otras empresas (Jorissen et al., 2005), sino también en etapas sucesivas, en las que las futuras generaciones, vinculadas a la gestión de la empresa, mantienen los valores asentados por la familia en etapas previas (Denison et al., 2004).

Sin embargo, a medida que la empresa se profesionaliza y su dirección pasa a manos de directivos no familiares contratados para la gestión empresarial, la familia pierde el control ejecutivo de la empresa y los nuevos directivos contratados, al no ser miembros de la familia, no necesariamente comparten los valores de la misma. Ellos son contratados para satisfacer los intereses de los propietarios (esto es, la revalorización de la organización - lograda con el crecimiento de la empresa - y la entrega de dividendos - única recompensa percibida por los propietarios familiares -) y evaluados por las decisiones que adopten en la empresa. Así, sus decisiones son más racionales y menos altruistas en comparación con las empresas de propiedad y dirección familiar, dado que con ellas se ha de buscar el logro de los intereses de los propietarios. Además, se trata de organizaciones de mayor tamaño y antigüedad (Gersick et al., 1997; Galve y Salas, 2003), perdiéndose así ese carácter familiar en favor de un carácter racional, ambiental o externo. Así, en estas organizaciones, a pesar de que en la cultura organizativa queden remanentes de la cultura familiar, se adopta una cultura más orientada al entorno.

En las empresas no familiares, los directores ejecutivos tienen un mayor grado de discrecionalidad en sus decisiones en comparación con los directivos de las empresas familiares (Schulze et al., 2001), al estar la propiedad diluida entre un grupo amplio de propietarios y soportar los propietarios un menor riesgo por su inversión, que está más diversificada (Anderson et al., 2002). Este mayor grado de discrecionalidad permite a los directivos actuar en su propio interés, para minimizar sus riesgos y buscar su beneficio, a costa de los intereses de los propietarios (Van den Berghe y Carchon, 2003), y buscar mayores salarios en la empresa, mayor estabilidad laboral, y mayor estatus y reconocimiento social, justificando todas sus acciones en el crecimiento empresarial, para así lograr que su satisfacción personal y la de los trabajadores de la empresa sea mayor (Werner et al., 2005). Para alcanzar estos propósitos es muy importante atender a las oportunidades del mercado, es por ello, que en estas organizaciones, se apuesta más que por el grupo, en el individuo, en su autonomía y capacidad de logro, así, como se da más importancia a las oportunidades del entorno que a la empresa. 
Por otro lado, el tamaño de estas organizaciones es significativamente mayor que en las empresas de propiedad y dirección familiar (Gersick et al., 1997; Galve y Salas, 2003), y los puestos de responsabilidad suelen estar ocupados durante un menor tiempo por los mismos directivos (Jorissen et al., 2005). Esto nos lleva a formular la primera proposición:

P1: La orientación a una cultura clan es alta en las empresas de propiedad y dirección familiar, media en las empresas familiares profesionalizadas y baja en las empresas no familiares.

\section{CULTURA DE MERCADO O RACIONAL}

Esta dimensión de la cultura organizativa se refiere a las creencias y a los valores de la empresa que están relacionados con el entorno, los objetivos, el logro, la competitividad, o el liderazgo de mercado (figura 1). Así, atendiendo a la argumentación anterior, en las empresas de propiedad y dirección familiar, a consecuencia de su estructura de propiedad, de su mayor aversión al riesgo, de su carácter no racional (altruismo), de su menor tamaño y recursos financieros, se espera una menor orientación al mercado que en las otras organizaciones.

Otros argumentos que soportan nuestras suposiciones en la cultura organizativa de la empresa de propiedad y dirección familiar son su orientación estratégica e internacionalizadora, y la formación de sus directivos. Con relación a la primera, Dockels y Frohlich (1991) encuentran que la orientación estratégica en las empresas de propiedad y dirección familiar es más conservadora y está menos orientada al crecimiento y a los beneficios que en las otras empresas. Es por tanto, una cultura menos orientada al mercado. En este sentido, Dyer (1986) afirma que las empresas de propiedad y dirección familiar son organizaciones que penetran en un nicho de mercado y permanecen en él.

Por otro lado, con relación al grado de internacionalización de la empresa, Gallo y Sveen (1991) sugieren que en la primera y segunda generación de estas empresas existe una menor orientación a la apertura a nuevos mercados. La expansión internacional y la orientación al mercado son decisiones arriesgadas, sobre todo en las fases iniciales del proceso, ya que se dispone de muy poca información (Fernández y Nieto, 2006). Por ello, si una empresa está concentrada en el mercado nacional, donde se encuentra bien integrada, con su cultura y necesidades, la expansión internacional se ve como una estrategia poco atractiva (Fernández y Nieto, 2005).

Por último, con relación a la formación de sus directivos, está suele ser baja por dos motivos. Primero, los miembros de la familia son quienes ocupan los puestos directivos de la empresa, y adoptan esta posición para evitar la pérdida del control ejecutivo de la empresa; ahora bien, es posible que no dispongan de suficientes familiares cualificados o con experiencia suficiente para comprender la dinámica del mercado (Fernández y Nieto, 2006). Además, el arraigo familiar impide que dichos familiares estén dispuestos a emprender acciones de mercado que puedan 
suponer costes a la familia, como abandonar su hábitat tradicional para emprender un proyecto internacional, esto es, la expatriación de familiares. Segundo, las empresas familiares encuentran problemas para atraer a directivos profesionales cualificados debido a la naturaleza poco estructurada de esas empresas (Schulze et al., 2001). Todas ellas, evidencias empíricas que nos muestran la menor orientación de las empresas de propiedad y dirección familiar al mercado.

En las empresas familiares profesionalizadas, la cultura organizativa es significativamente diferente a la de las empresas de propiedad y dirección familiar y está más orientada al mercado, al perder la familia el control en la dirección de la empresa, al no poder observar directamente los comportamientos de los miembros de la empresa y al suplir tal pérdida por un sistema de incentivos con el que vigilar sus actuaciones, en base a objetivos que corrijan y alineen las acciones de la empresa con los intereses de los propietarios (Alchian y Demsetz, 1972). Además, en las empresas familiares profesionalizadas, existe una mayor formación de sus directivos, lo que les permite comprender mejor al entorno y posicionar mejor en él a la organización. Sin embargo, en dicho posicionamiento, los directivos de estas empresas adoptan en dicho proceso unas posturas menos arriesgadas en el mercado, lo que hará que dichas organizaciones tengan una menor orientación al mercado que las empresas no familiares. Este comportamiento viene determinado por el hecho de sus directivos están sujetos a mayores riesgos que los directivos de las empresas no familiares, al estar más controladas sus decisiones por los propietarios familiares y más expuestos a un despido laboral en caso de adoptar una decisión errónea.

Por último, en las empresas no familiares, al disponer sus directivos de un mayor grado de discrecionalidad, éste puede ser aprovechado para emprender acciones que satisfagan a los miembros de la empresa y no a los propietarios. Entre ellas, Werner et al. (2005) señalan la estrategia de crecimiento de la empresa en el mercado, como medida para justificar un mayor salario organizativo, y una mayor satisfacción personal de los directivos. Esto nos lleva a plantear la segunda proposición:

P2: La orientación a una cultura de mercado es alta en las empresas no familiares, media en las empresas familiares profesionalizadas y baja en las empresas de propiedad y dirección familiar.

\section{CULTURA DE ADHOCRACIA O CAMBIO}

Esta tercera dimensión de la cultura organizativa refleja las creencias y valores relacionados con la actitud emprendedora, el liderazgo de productos, la adquisición de productos, la innovación y la asunción de riesgos (figura 1). Esta dimensión ha sido ampliamente examinada en la literatura de empresa familiar - Zahra et al. (2004), Gudmundson et al. (2003), Hall et al. (2001) y Pistrui et al. (2001) -, aunque la evidencia empírica previa no es totalmente coincidente, ni concluyente. No obstante, en un gran número de trabajos recientes se sostiene el mayor carácter 
emprendedor de las empresas de propiedad y dirección familiar (Zahra et al., 2004), motivado por la existencia de diversos valores favorecedores del cambio como los valores de grupo o equipo que en conjunto compensan valores negativos de la cultura de estas empresas como la menor orientación a los valores de mercado. Chandler et al. (2000) sostienen estos argumentos, al encontrar que la mayor cultura innovadora se da en las empresas más pequeñas, en las que existen unas prácticas de recursos humanos menos formalizadas, y unos peores recursos organizativos, aspectos que caracterizan más a las empresas de propiedad y dirección familiar que a las otras empresas. Ogbonna y Harris (2000) afirman que existe una mayor tendencia a la innovación en aquellas empresas menos burocratizadas, en las que existe un liderazgo participativo. Este es el caso de las empresas de propiedad y dirección familiar donde el liderazgo se ejerce participativamente a través de los miembros de la familia, y en ocasiones esos lazos familiares se extienden a otros miembros de la organización (Zahra et al., 2004). Además, en la empresas familiares, tanto en las de propiedad y dirección familiar como en las familiares profesionalizadas, se invierte más en recursos intangibles como la reputación, la imagen de marca y en el liderazgo de productos (Nieto, 2003), al estar tales recursos vinculados en muchas ocasiones al apellido familiar. Por último, en las organizaciones de propiedad y dirección familiar, la figura del fundador ha estado siempre asociada a la de una persona altamente emprendedora (Hall et al., 2001).

En las empresas familiares profesionalizadas, no existe evidencia empírica previa que caracterice a la cultura adhocrática en tales empresas. De hecho, no existen trabajos que relacionen a la cultura del cambio con la estructura de propiedad de la empresa (Gudmunson et al., 2003). Pero, partiendo de los argumentos anteriores, se espera que en estas organizaciones existan unos valores más orientados al grupo que en las empresas no familiares, aunque unos valores menos orientados al mercado. Y partiendo de la idea de que en dichas organizaciones, pese a que la familia ya no forma parte de los órganos de gestión en la empresa, si lo hace en los de gobierno, adoptando desde allí las principales decisiones estratégicas de la empresa. Esa implicación familiar, conducirá a una cultura más orientada al cambio que en las empresas no familiares, donde los propietarios dispondrán de un menor poder de control de la gestión de la empresa y los directivos serán adversos a la asunción de riesgos personales en la empresa. Es decir, los directivos de las empresas no familiares, aprovechando su mayor capacidad decisora en la empresa (si la comparamos con la de los directivos de las empresas familiares profesionalizadas) evitaran toda situación de riesgo personal y toda situación de cambio en la organización que generé incertidumbre sobre sus intereses. Es por ello que planteamos la tercera proposición:

P3: La orientación a una cultura de adhocracia es alta en las empresas de propiedad y dirección familiar, media en las empresas familiares profesionalizadas y baja en las empresas no familiares. 


\section{CULTURA DE JERARQUÍA}

Esta última dimensión de la cultura se relaciona con los valores de seguridad, reglas formales, control, estructura, coordinación y eficiencia interna (figura 1). Atendiendo a las argumentaciones anteriormente desarrolladas para las otras tres dimensiones culturales, en las empresas de propiedad y dirección familiar se esperan unos valores menos orientados a la jerarquía, y es que a pesar de su estructura de propiedad, por el que el poder de decisión de la familia está centrado en la empresa, ese gran control no se traduce en un gran uso de políticas formales muy estructuradas (Lyman, 1991). Además, el comportamiento de los trabajadores puede controlarse mediante sistemas formales de supervisión o a través de incentivos. En las empresas no familiares, y atendiendo a los intereses de los directivos, señalados anteriormente, se preferirán remuneraciones salariales fijas porque cubren mejor sus intereses (Werner et al., 2005). Sin embargo, en los dos tipos de empresas familiares, para evitar las acciones que beneficien unilateralmente a los directivos y empleados, y para buscar situaciones que beneficien a todos (Werner et al., 2005), se preferirán los sistemas de incentivos en la empresa, dado que hace partícipes del riesgo de la familia a todos los miembros de la empresa (Baker et al., 1988). Además, en situaciones, donde es difícil supervisar a los empleados, bien porque la empresa es grande, bien porque las tareas son complejas, los sistemas de incentivos también serán más adecuados (Jensen y Murphy, 1990).

Por otro lado, también existen estudios que confirman lo anterior. Es decir, en la mayoría de empresas de propiedad y dirección familiar se pretende lograr un elevado grado de fidelidad y satisfacción de sus trabajadores, clientes y proveedores (Donckels y Fröhlich, 1991), y esto no se lograría a través de organizaciones excesivamente burocratizadas y formalizadas. Se trata de organizaciones con menores recursos financieros y humanos, por lo que en ellas desarrollar unas estructuras altamente jerarquizadas no sería eficiente y además, estas desventajas en recursos son afrontadas por las empresas desarrollando estructuras menos burocratizadas y más flexibles. Denison et al. (2004) sostienen que en la cultura de las empresas de propiedad y dirección familiar se promulga más la adaptabilidad de la empresa.

En las empresas familiares profesionalizadas se desarrollaran valores culturales más orientados a la jerarquía, al tratarse de organizaciones más complejas que las de propiedad y dirección familiar, y al estar dirigidas por profesionales mejor preparados, capaces de instaurar en la empresa sistemas organizativos más profesionalizados. Aunque esta orientación será menor que en las no familiares, donde la tendencia al crecimiento convertirá a estas organizaciones en las más burocratizadas. Esto nos lleva a plantear la cuarta proposición:

P4: La orientación a la cultura de jerarquía es alta en las empresas no familiares, media en las empresas familiares profesionalizadas y baja en las empresas de propiedad y dirección familiar. 


\section{CONCLUSIONES}

El propósito de este estudio ha sido caracterizar la cultura organizativa en la empresa familiar, utilizando para ello, el instrumento de medida de la cultura propuesto por Cameron y Quinn (1999). Se plantea en el trabajo que la cultura organizativa varía entre las empresas, e influye de forma distinta en sus resultados, siendo por tanto, un recurso exclusivo con el que cada una de las organizaciones son capaces de desarrollar y mejorar sus ventajas competitivas (Zahra et al., 2004). Esta idea contribuye a mejorar la comprensión de las organizaciones $\mathrm{y}$, en concreto, entender mejor como el grado de concentración de la propiedad y la composición de los órganos de dirección afectan a los recursos de la empresa, bien entendiendo mejor las decisiones de los propietarios y directivos en la empresa y como estas afectan a los recursos y resultados organizativos, bien para caracterizar mejor a la organización.

Específicamente, se ha planteado que en las empresas familiares se da una mayor orientación a la cultura clan, mientras que en las empresas no familiares predominan las culturas de mercado y jerárquica.

En segundo lugar, la cultura organizativa en la empresa familiar queda marcada fuertemente por el fundador $\mathrm{y}$, pese a la profesionalización, los valores ya instaurados suelen seguir vigentes en futuras generaciones, aunque estas se desvinculen de la organización (Denison et al., 2004). Una última explicación reside en el hecho de que la cultura es un recurso estratégico, y como tal, será decidida desde los órganos de gobierno de la empresa. En empresas de propiedad y dirección familiar y en empresas familiares profesionalizadas dichos órganos estarán ocupados en mayor o menor medida por miembros de la familia, los cuales trasladarán sus valores a las estrategias generales organizativas (Zahra et al., 2004).

Este estudio defiende la validez de la teoría de recursos y capacidades, al examinar como varían los recursos de la empresa, en función del tipo de organización, atendiendo al grado de propiedad en la empresa y a la estructura de los órganos de dirección. Dado que desde esa teoría, la cultura organizativa es un recurso intangible y estratégico, crítico para los resultados de la organización (Zahra et al., 2004; Barney, 1986), se ha defendido como la cultura organizativa en la empresa familiar es un recurso difícil de modificar, es decir, la cultura generada por el fundador en la empresa familiar, pese a que la familia se desvincule de la dirección de la empresa, se mantiene en la organización. Estas relaciones entre resultados y recursos, pese a que han sido tratadas en la literatura, necesitan ser examinadas más exhaustivamente desde recursos y capacidades, matizando mejor la influencia entre cultura organizativa y otros recursos en la organización, así como las ventajas generadas de tales relaciones.

Desde una visión práctica, los directivos de las organizaciones, deberían saber identificar la cultura de su empresa, comprenderla, y aprovechar los efectos positivos que pueden obtenerse de tales recursos. Su estudio, permitiría a la 
familia, conocer su grado de influencia en la empresa, así como valorar si tal influencia concuerda favorablemente o no con otros recursos de la organización. Es, por tanto, un recurso que debería de examinarse mejor por gestores y propietarios en las organizaciones.

\section{BIBLIOGRAFÍA}

ALCHIAN. A. y DEMSETZ., H. (1972), Production, Information Costs, and Economic Organization, American Economic Review, Vol. 62(5), 777-795.

ALVESSON, M. (1993), Cultural perspectives on organizations, University Press, Cambridge.

AMIT, R. y SCHOEMAKER, P.J. (1993), Strategic assets and organizational rent, Strategic Management Journal, Vol. 14 (1), 33-46.

ANDERSON, R., MANSI, S. y REEB D (2002), Founding family ownership and the agency cost of debt, Journal of Financial Economics, Vol. 68 (2), 263285.

BAKER, G., JENSEN, M. y MURPHY, K (1988), Compensation and incentives: Practice vs. Theory, Journal of Finance, Vol. 18 (3), 593-616.

BARNEY, J.B. (1986), Organizational culture: Can it be a source of sustained competitive advantage?, Academy of Management Review, Vol. 11(3), 656665 .

BARNEY, J.B. Firm resources and sustained competitive advantage. Journal of Management, Vol.17, n'.1, 1991, p. 99-120.

BARONTINI, R. y CAPRIO, L. (2006), The Effect of Family Control on Firm Value and Performance: Evidence from Continental Europe, European Financial Management, Vol. 12 (5),. 689-723.

CAMERON, K. y QUINN, R. (1999), Diagnosing and Changing Organizational Culture. Addison-Wesley Series, New York.

CHANDLER, G., KELLER, C. y LYON, D. (2000), Unraveling the Determinants and Consequences of an Innovation-Supportive Organizational Culture, Entrepreneurship Theory and Practice, Vol. 25(1), 59-76.

CHRISMAN, J.J., CHUA, J. y STEIER, L. (2002), The influence of national culture and family involvement on entrepreneurial perceptions and performance at the state level, Entrepreneurship Theory and Practice, Vol. 26(4), 113-130.

CORBETTA, G. y MONTEMERLO, D. (1999), Ownership, governance, and management issues in small and medium-size family businesses: A 
comparison of Italy and the United States, Family Business Review, Vol. 12 (4), 361-374.

DE LONG, D.W. y FAHEY, L. (2000), Diagnosing cultural barriers to knowledge management, Academy of Management Executive, Vol. 14(4), 113-127.

DENISON, D. (1990), Corporate culture and organizational effectiveness, wILEY, New York.

DENISON, D., LIEF, C. y WARD, J. (2004), Culture in Family-Owned Enterprises: Recognizing and Leveraging Unique Strengths, Family Business Review, Vol. 17 (1), 61-71.

DENISON, D.R. y SPREITZER, G. (1991). En WOODMAN, R. W. y PASSMORE, W. A. Research in Organizational Change and Development, Greenwich: Ct: JAI Press, 1991, pp. 1-21.

DETERT, J.R., SCHROEDER, R.G. y MAURIEL, J.J. (2000), A framework for linking culture and improvement initiatives in organizations, Academy of Management Review, Vol. 25(4), 850-863.

DIERICKX, I. y COOL, K. (1989), Asset stock accumulation and sustainability of competitive advantage, Management Science, Vol. 35(12), 1504-1511.

DONCKELS, R. y FRÖLICH, E. (1991), Are your Family Businesses Really Different? European Experiences from STRATOS, Family Business Review, Vol. 4 (2), 149-160.

DYER, W.G. (1986), Cultural Change in Family Firms: Anticipating and Managing Business and Family Transitions, Jossey-Bass, San Francisco.

FERNÁNDEZ, Z. y NIETO, M.J. (2005), La estrategia de internacionalización de la pequeña empresa familiar, Cuadernos de Economía y Dirección de la Empresa, Vol. 22, 107-125.

FERNÁNDEZ, Z. y NIETO, M.J (2006), Internationalization Strategy of Small and Medium-Sized Family Businesses: Some Influential Factors, Family Business Review, Vol. 18(1), 77-90.

GALLO, M. A. y SVEEN, J. (1991), Internationalizing the family business: Facilitating and restraining factors, Family Business Review, Vol. 4(2),181190.

GALLO, M., TÀPIES, J. y CAPPUYNS, K. (2004), Comparison of Family and Nonfamily Business, Family Business Review, Vol. 17(4), 303-318.

GALVE, C. y SALAS, V (2003), La empresa familiar en España, Fundación BBVA, Bilbao. 
GERSICK, E., DAVIS, J.A., McCOLLOM, M. y LANSBERG, I. (1997), Empresas familiares. Generación a generación, McGraw Hill, Mexico.

GÓMEZ-MEJÍA, L.R.,; NUÑEZ-NICKEL, M. y GUTIÉRREZ, I. (2001), The role of family ties in agency contracts, Academy of Management Journal, Vol. 44 (1), 81-95.

GRANT, R.M. (1996), Dirección Estratégica: Conceptos, Técnicas y Aplicaciones, Cívitas, Madrid:.

GUDMUNDSON, D., BURK TOWER, C. y HARTMAN, E.A. (2003), Innovation in small businesses: Culture and ownership structure do matter, Journal of Developmental Entrepreneurship, Vol. 8(1), 1-17.

HALL, A., MELIN, L. y NORDQVIST, M. (2001), Entrepreneurship as Radical Change in the Family Business: Exploring the Role of Cultural Patterns, Family Business Review, Vol. 14(3), 193-208.

JENSEN, M.C. y MURPHY, K.J. (1990), Performance pay and top-management incentives, Journal of Political Economy, Vol. 98(2), 225-264.

JORISSEN, A., LAVERON, E., MARTENS, R. y REHEUL, A. (2005), Real Versus Sample-Based Differences in Comparative Family Business Research, Family Business Review, Vol. 18(3), 229-247.

KARRA, N., TRACEY, P. y PHILLIPS, N. (2006), Altruism and Agency in the Family Firm, Entrepreneur Theory and Practice, Vol. 30(6), 861-877.

KETS DE VRIES, M.F.R. (1993), The dynamics of family controlled firms: the good and the bad news, Organizational Dynamics, Vol. 21(3), 59-71.

KOTTER, J., y HESKETT, J. (1992), Corporate Culture and Performance, Free Press, New York.

LYMAN, A. (1991), Customer service: Does family ownership make a difference?, Family Business Review, Vol. 4 (3), 303-324.

NIETO, M.J. (2003), Las pyme familiares en España. ¿Qué nos dicen los datos?, Investigaciones Europeas de Dirección y Economía de la Empresa, Vol. 9(2), 115-128.

OGBONNA, E., y HARRIS, L. (2000), Leadership Style, Organizational Culture and Performance: Empirical Evidence from UK Companies, International Journal of Human Resource Management, Vol. 11(4), 766-788.

ORTEGA PARRA, A. y SASTRE CASTILLO, M.A. (2013), Impact of perceived corporate culture and organizational commitment, Management Decision, Vol. 51 (5), 1071-1083. 
PISTRUI, D., HUANG, W., OKSOY, D., JING, Z., y WELSCH, H. (2001), Entrepreneurship in China: Characteristics, attributes, and family forces shaping the emerging private sector, Family Business Review, Vol. 14 (2), $141-152$.

POZA, E.J., ALFRED, T. y MAHESWARI, A. (1997), Stakeholder perceptions of culture and management practices in family and family firms. A preliminary report, Family Business Review, Vol. 10 (2), 135-155.

QUINN, R.E.(1988), Beyond Rational Management: Mastering the Paradoxes and Competing Demands of High Performance, Jossey-Bass, San Francisco.

QUINN, R.E. y SPREITZER, G.M. (1991), The psychometrics of the Competing Values Culture Instrument and an analysis of the impact of organizational culture on quality of life. En WOODMAN, R.W. y PASMORE, W.A. Research in organizational change and development, Greenwich, CT: JAI Press.

ROGOFF, E.G. y HECK, R.K.Z. (2003), Evolving research in entrepreneurship and family business: Recognizing family as the oxygen that feeds the fire of entrepreneurship, Journal of Business Venturing, Vol. 18 (5), 559-566.

SANCHEZ MARÍN, G., DANVILA DEL VALLE, I. y SASTRE CASTILLO, M.A. (2015), Entrepreneurship and Family Business: does the organizational culture affect to firm performance?. En PERIS ORTIZ, M. y SAHUT, J.M. New challenges in entrepreneurship and finance, Paris: Springer, 169-179.

SCHEIN, E. H. (1995), The role of the founders in creating organizational culture, Family Business Review, Vol. 8 (3), 221-238.

SCHULZE, W., LUBATKIN, M., DINO, R., y BUCHHOLTZ, A. (2001), Agency relationships in family firms: Theory and evidence, Organization Science, Vol. 12 (2), 99-116.

SCHULZE, W.S., LUBATKIN, M.H., y DINO, R.N. (2003), Exploring the agency consequences of ownership dispersion among the directors of private family firms, Academy of Management Journal, Vol. 46 (2), 179-194.

SORENSON, E.D. (2000), The Contribution of Leadership Style and Practices to Family and Business Success, Family Business Review, Vol. 13(3), 183-200.

STEIER, L. (2001), Family firms, plural forms of governance, and the evolving role of trust, Family Business Review, Vol. 14(4), 353-367.

STOCK, G., McFADDEN, K. y GOWEN III, C. (2007), Organizational culture, critical success factors, and the reduction of hospital errors, International Journal or Production Economics, Vol. 106(2), 368-392. 
VAN DEN BERGHE, L. y CARCHON, S. (2003), Agency relations within the family business system: An exploratory approach, Corporate Governance, Vol. 11(3), 155-285.

WERNER, S., TOSI, H. y GOMEZ-MEJIA, L.R (2005), Organizational governance and employee pay: How ownership structure affects the firm's compensation strategy, Strategic Management Journal, Vol. 26(4), 377-384.

ZAHRA, S., HAYTON, J. y SALVATO, C. (2004), Entrepreneurship in Family vs. Non-Family Firms: A Resource Based Analysis of the Effect of Organizational Culture, Entrepreneurship Theory and Practice, Vol. 28(4), 363-381. 